\title{
Diazepam-binding inhibitor mediates feedback regulation of pancreatic secretion and postprandial release of cholecystokinin
}

\author{
Ying Li, Yibai Hao, and Chung Owyang \\ Gastroenterology Research Unit, Department of Internal Medicine, University of Michigan Health System, \\ Ann Arbor, Michigan 48109, USA \\ Address correspondence to: Chung Owyang, 1500 East Medical Center Drive, University of Michigan Health System, \\ Ann Arbor, Michigan 48109-0362, USA. Phone: (734) 936-4785; Fax: (734) 936-7392; E-mail: cowyang@umich.edu.
}

Received for publication April 28, 1999, and accepted in revised form December 20, 1999.

Recently, we isolated a trypsin-sensitive cholecystokinin-releasing peptide (CCK-RP) from porcine and rat intestinal mucosa. The amino acid sequence of this peptide was determined to be identical to that of the diazepam-binding inhibitor (DBI). To test the role of DBI in pancreatic secretion and responses to feeding, we used pancreaticobiliary and intestinal cannula to divert bile-pancreatic juice from anesthetized rats. Within 2 hours, this treatment caused a 2 -fold increase in pancreatic protein output and a >10-fold increase in plasma CCK. Luminal DBI levels increased 4-fold. At 5 hours after diversion of bile-pancreatic juice, each of these measures returned to basal levels. Intraduodenal infusion of peptone evoked a 5 -fold increase in the concentration of luminal DBI. In separate studies, we demonstrated that intraduodenal administration of antiserum to a DBI peptide specifically abolished pancreatic secretion and the increase in plasma CCK levels after diversion of bile-pancreatic juice. To demonstrate that DBI mediates the postprandial rise in plasma CCK levels, we showed that intraduodenal administration of 5\% peptone induced dramatic increases in pancreatic secretion and plasma CCK, effects that could be blocked by intraduodenal administration of anti-DBI antiserum. Hence, DBI, a trypsin-sensitive CCK-RP secreted from the proximal small bowel, mediates the feedback regulation of pancreatic secretion and the postprandial release of CCK.

J. Clin. Invest. 105:351-359 (2000).

\section{Introduction}

Feedback regulation of pancreatic secretion has been demonstrated clearly in the rat $(1,2)$. Intraduodenal proteases such as trypsin or chymotrypsin inhibit pancreatic secretion, whereas protease inhibitors, intact protein, or pancreatic juice diverted from the proximal small intestine stimulate pancreatic secretion. A similar feedback control system has been shown to operate in chickens (3), pigs (4), and in humans (5). The feedback regulation of pancreatic secretion by trypsin is mediated by the release of cholecystokinin (CCK) (6). Two groups of investigators have demonstrated in rats that stimulation of pancreatic secretion and CCK release after diversion of pancreatic juice is induced by a trypsin-sensitive CCK-releasing peptide (CCK-RP) that is secreted into the intestinal lumen ( 7 , 8). Experimental evidence suggests that intact protein, such as casein, acts by competing for proteolytic enzymes that would otherwise inactivate the CCK-RP. The resulting increase of active CCK-RP in the intestine induces the release of CCK and stimulates pancreatic secretion. In contrast, peptone, a pancreatic protease digest of protein, can stimulate the secretion of CCK-RP (9). The concerted action of these two mechanisms may be important in the postprandial increase of CCK levels.

We recently isolated a trypsin-sensitive CCK-RP from porcine and rat intestinal mucosa (10). This peptide has a molecular weight of $9.81 \mathrm{kDa}$ and is composed of 89 amino acids that are identical in sequence to the amino acids of the diazepam-binding inhibitor (DBI). At the same time, another group purified a luminal CCKreleasing factor from rat intestinal secretions (11). We hypothesized that DBI is the CCK-RP responsible for the feedback regulation of pancreatic secretion and the postprandial release of CCK. To support this hypothesis we needed to demonstrate that DBI fulfills the following criteria: (a) intraduodenal administration of DBI should increase pancreatic secretion and plasma CCK levels; (b) increases in luminal DBI immunoreactivities should parallel changes in plasma CCK levels during diversion of pancreatic juice and during intraduodenal peptone stimulation; and (c) intraduodenal administration of DBI antiserum should abolish pancreatic secretion and release of CCK during diversion of bile-pancreatic juice and during peptone stimulation.

\section{Methods}

Materials. Soybean trypsin inhibitor (SBTI), peptone (enzymatic hydrolysate from meat, type I), casein, and maltose were purchased from Sigma Chemical Co. (St. Louis, Missouri, USA). L364,718 was a gift from Victor Lotti, Merck Sharp and Dohme, West Point, Pennsylvania, USA. $\mathrm{DBI}_{33-50}$ and $\mathrm{DBI}_{1-86}$ were synthesized by the University of Michigan Protein and Carbohydrate Structure Facility (Ann Arbor, Michigan, USA). 
Animal preparation. Male Sprague-Dawley rats, weighing between 250 and $300 \mathrm{~g}$, were fasted overnight and anesthetized with a mixture of xylazine and ketamine (13 and $87 \mathrm{mg} / \mathrm{kg}$ body weight, respectively). Supplemental doses were given every 1.5 hours as needed to maintain adequate anesthesia. A polyethylene catheter was inserted through a midline incision into the common bile pancreatic duct at the ampulla of Vater. A second catheter was placed in the duodenum, slightly above the sphincter of Oddi for intestinal perfusion of pancreaticobiliary juice. The abdominal wound was covered with saline-moistened gauze, and the animal was maintained at $36^{\circ} \mathrm{C}$ with a heating pad. After a 30minute stabilization period, pancreaticobiliary secretions were collected every 15 minutes. The volume was measured, and an aliquot was taken and diluted with distilled water for protein determination. The remainder of the undiluted bile-pancreatic juice was returned to the intestine through the duodenal cannula during the next collection period. Protein in the bile-pancreatic juice was measured spectrophotometrically using the assay method of Bradford. Our previous studies have confirmed that the increased levels of protein in the bile-pancreatic juice after CCK-8 stimulation were mainly from the pancreatic source. CCK stimulation did not affect biliary protein levels.

Effects of DBI $I_{33-50}$ on plasma CCK levels and pancreatic secretion (intraluminal DBI ${ }_{33-50}$ dose-response studies). To investigate the effect of intraluminal administration of $\mathrm{DBI}_{33-50}$ on plasma CCK levels and pancreatic secretion, we used the anesthetized rat model with bile-pancreatic juice diversion as described previously (12). After a 30-minute basal period, bile-pancreatic juice was diverted, and the duodenum was perfused with SBTI (10 mg/kg, dissolved in $0.5 \mathrm{~mL}$ saline, given as a bolus) and then with normal saline at $1 \mathrm{~mL} / \mathrm{h}$ for 5 hours. Pancreatic secretion was collected every 15 minutes. After a 5-hour diversion of bile-pancreatic juice, an intraduodenal injection of graded doses of synthetic porcine $\mathrm{DBI}_{33-50}(0.5-1000 \mu \mathrm{g}$, dissolved in saline) was administered over 5 minutes. Pancreatic protein output was monitored every 15 minutes after the injection. CCK levels were measured in blood samples obtained by cardiac puncture 45 minutes after the infusion of $\mathrm{DBI}_{33-50}$, as described previously (6).

Effects of DBI ${ }_{33-50}$ on plasma CCK levels and pancreatic secretion in response to a casein meal. To demonstrate that DBI stimulates CCK release and pancreatic enzyme secretion postprandially, we investigated the effect of $\mathrm{DBI}_{33-50}$ on the duodenal infusion of casein, a protein that is known to elevate plasma CCK levels and pancreatic secretion because it inhibits trypsin activity and activation of the feedback loop (13). Twelve percent casein was infused into the duodenum through a cannula at a rate of 3 $\mathrm{mL} / \mathrm{h}$ for 2 hours. Pancreatic secretion was collected every 15 minutes as described previously. The study was performed in 2 groups of rats: (a) those who received an intraduodenal injection of $\mathrm{DBI}_{33-50}(10 \mu \mathrm{g})$ at 1 hour after casein infusion and (b) those pretreated with the
CCK antagonist L364,718 $(0.5 \mathrm{mg} / \mathrm{kg})$, given intravenously as a bolus, 10 minutes before casein infusion. L364,718 was dissolved in 1:1 DMSO/polysorbate 80. The solution was diluted with $0.9 \%$ saline to a final concentration of $4 \% \mathrm{DMSO} /$ polysorbate 80 .

Luminal secretion of DBI. To determine if DBI is released during diversion of bile-pancreatic juice, we measured luminal DBI immunoreactivity at different time points after the diversion. Anesthetized rats were prepared with pancreaticobiliary cannula. A 24-cm segment of small intestine including the whole duodenum and the proximal jejunum was isolated between 2 cannulae: one positioned at $4 \mathrm{~cm}$ (PE 60; $0.76 \mathrm{~mm}$ inner diameter, 1.22 $\mathrm{mm}$ outer diameter) from the ligament of Treitz for the infusion of saline or nutrient solution and the other at $24 \mathrm{~cm}$ (PE 190; $1.19 \mathrm{~mm}$ inner diameter, $1.7 \mathrm{~mm}$ outer diameter) from the ligament of Treitz for collection of intestinal perfusates. After a 30-minute basal period, rats received an intraduodenal perfusion of PBS (20
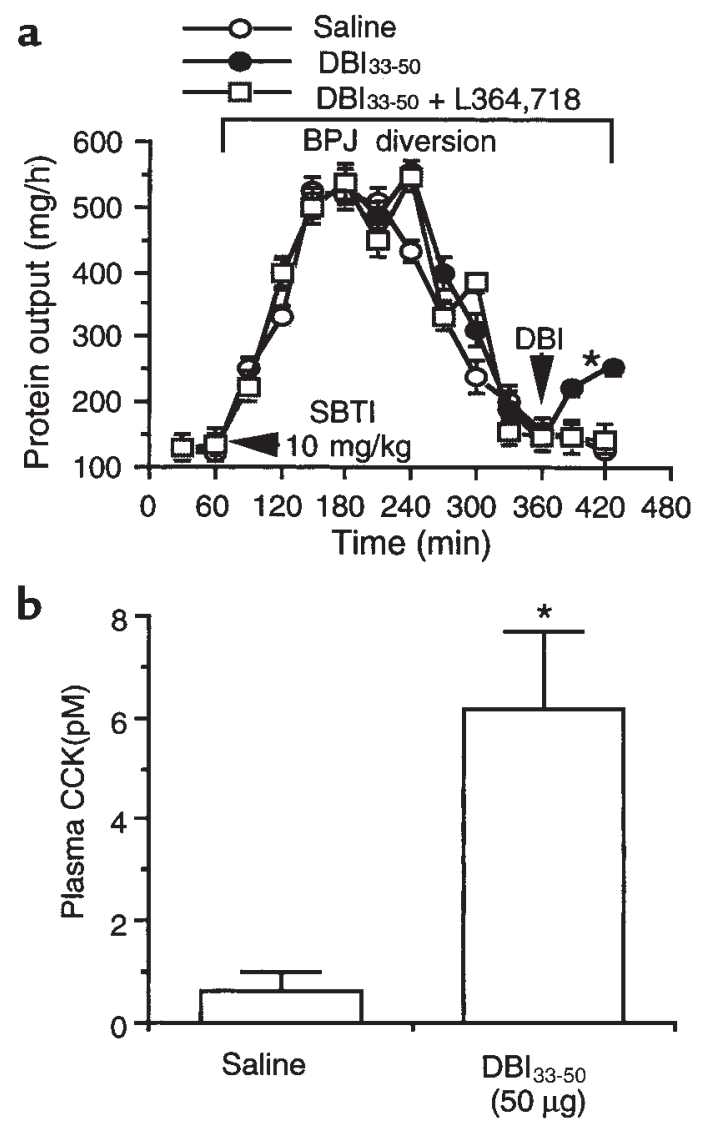

Figure 1

Effect of intraduodenal administration of $\mathrm{DBI}_{33-50}(50 \mu \mathrm{g})$ on pancreatic protein and plasma CCK levels in rats after a 5-hour diversion of bile-pancreatic juice (BPJ). (a) Pancreatic protein outputs after the administration of $\mathrm{DBI}_{33-50}$ (from 330 to 420 minutes) in the presence or absence of $\mathrm{L} 364,718$ were compared by ANOVA (total degrees of freedom, 71; $F=182.39 ; P<0.01$. (b) Plasma CCK levels were compared by Student's $t$ test, $P<0.01$. Values are means $\pm \mathrm{SE}$ for 6 rats in each group. Administration of the CCK-A receptor antagonist $\mathrm{L} 364,718$ prevented the increase in protein secretion stimulated by $\mathrm{DBI}_{33-50}$. 


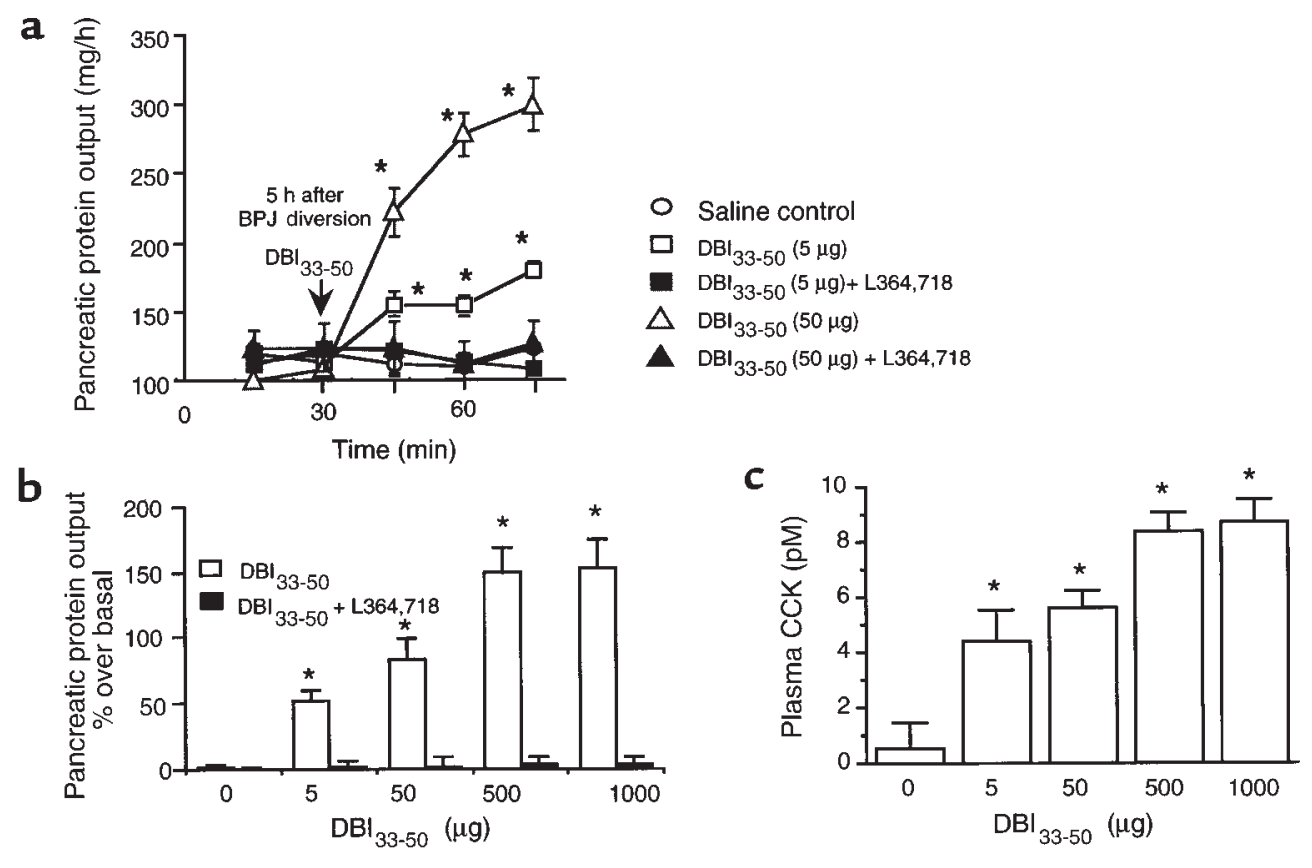

Figure 2

(a) Pancreatic protein output after administration of $\mathrm{DBI}_{33-50}$ at 5 hours after BPJ diversion. (ANOVA total degree of freedom, 149; $F=103.59$; $P<0.01)$. (b) Intraduodenal administration of $\mathrm{DBI}_{33-50}$ after a 5-hour diversion of BPJ elicits dose-dependent increases in pancreatic protein secretion. Values are mean $\pm S E$ for 6 rats in each group. (ANOVA, total degrees of freedom, 59; $F=2128.3 ; P<0.01$.) (c) Plasma CCK levels in response to intraduodenal administration of $\mathrm{DBI}_{33-50}$. Values are mean $\pm \mathrm{SE}$ for 6 rats in each group. Student's $t$ test, $P<0.01$. Note that in b and $\mathbf{c}$ pancreatic protein outputs and plasma CCK levels were measured at 45 minutes after the administration of DB| $\left.\right|_{33-50}$.

$\mathrm{mL} / 10 \mathrm{~min})$. DBI-like immunoreactivity in the perfusates collected was measured to determine the luminal DBI concentrations under basal conditions. In a separate group of rats, perfusates were obtained after a 2-hour and a 5-hour diversion of bile-pancreatic juice. In previous studies, we used the donor-recipient rat model to demonstrate that peptone and oleic acid stimulate pancreatic protein secretion and CCK release in a manner that parallels the changes in luminal CCK-RP levels $(9,14)$. To demonstrate that nutrient stimulates DBI secretion, $5 \%$ peptone was perfused for 1 hour after a 5-hour diversion of bile-pancreatic juice when plasma CCK concentrations had returned to basal levels. The intestinal perfusates collected from the rats were filtered with Whatman No. 54 paper (Whatman International Ltd., Maidstone, England) to remove any large particles and intestinal debris. The filtrate was ultrafiltered with an Amicon membrane (YM1; Amicon, Beverly, Massachusetts, USA), and the residue, which contained DBI (mol wt $>1000$ ), was dissolved in water and lyophilized. DBI-like immunoreactivity in the concentrated perfusates was measured by RIA using an antibody against porcine $\mathrm{DBI}_{33-50}$ (see next section). Plasma CCK levels were measured after a 2 -hour and a 5 -hour diversion of bile-pancreatic juice and after a 1-hour infusion of 5\% peptone as described previously (6).

Generation of anti-DBI antisera. Porcine $\mathrm{DBI}_{33-50}(1 \mathrm{mg})$ was dissolved in $1 \mathrm{~mL}$ of PBS, emulsified with $1 \mathrm{~mL}$ Freund's complete adjuvant, and injected subcutaneously into 4 New Zealand rabbits $(1-1.5 \mathrm{~kg})$. Each animal received 6-8 injections per week. Six weeks after the primary immunization, blood was collected and serum was separated after each immunization to monitor the antibody titer by ${ }^{125}$ I-DBI-binding studies. The collected sera were diluted with equal volumes of glycerol and stored at $-80^{\circ} \mathrm{C}$. To increase the antibody titer, intramuscular injections of the same antigen dose emulsified in Freund's incomplete adjuvant were administered every 2 weeks for 3 months. The final antibody titer was 1:40,000. This antibody showed complete cross-reactivity with porcine $\mathrm{DBI}_{1-86}$ and $\mathrm{DBI}_{33-50}$ but has no crossreactivity with secretin, CCK, vasoactive intestinal polypeptide (VIP), or somatostatin. It has an $\mathrm{IC}_{50}$ of 9 $\mathrm{pM}$ and $7 \mathrm{pM}$ for $\mathrm{DBI}_{1-86}$ and $\mathrm{DBI}_{33-50}$, respectively.

RIA of DBI. An $100 \mu \mathrm{L}$ of the lyophilized intestinal perfusates collected from each sample was incubated with $100 \mu \mathrm{L}$ of rabbit anti-serum for 24 hours at $4^{\circ} \mathrm{C}$. After the incubation, each sample was incubated with $100 \mu \mathrm{L}$ of ${ }^{125} \mathrm{I}_{-\mathrm{Tyr}}-\mathrm{DBI}_{33-50}(0.012 \mu \mathrm{Ci}$ per tube) for 24 hours at $4^{\circ} \mathrm{C}$. The radiolabeled porcine $\mathrm{DBI}_{33-50}$ was prepared by the Bolton-Hunter method and was dissolved in physiological buffer containing $0.1 \mathrm{M}$ sodium phosphate $(\mathrm{pH}$ 7.4), $0.05 \mathrm{M} \mathrm{NaCl}, 0.1 \% \mathrm{BSA}$, and $0.01 \%$ sodium azide. The goat anti-rabbit $1 \mathrm{gG}$ serum $(100 \mu \mathrm{L})$ and normal rabbit serum $(100 \mu \mathrm{L})$ were then added and incubated for 90 minutes at $24^{\circ} \mathrm{C}$. All these reagents were reconstituted using the RIA buffer. Finally, $500 \mu \mathrm{L}$ of the RIA buffer was added and centrifuged at approximately 1700 $g$ for 20 minutes at $4^{\circ} \mathrm{C}$. The supernatant was completely aspirated, and the radioactivity of the pellet was count- 

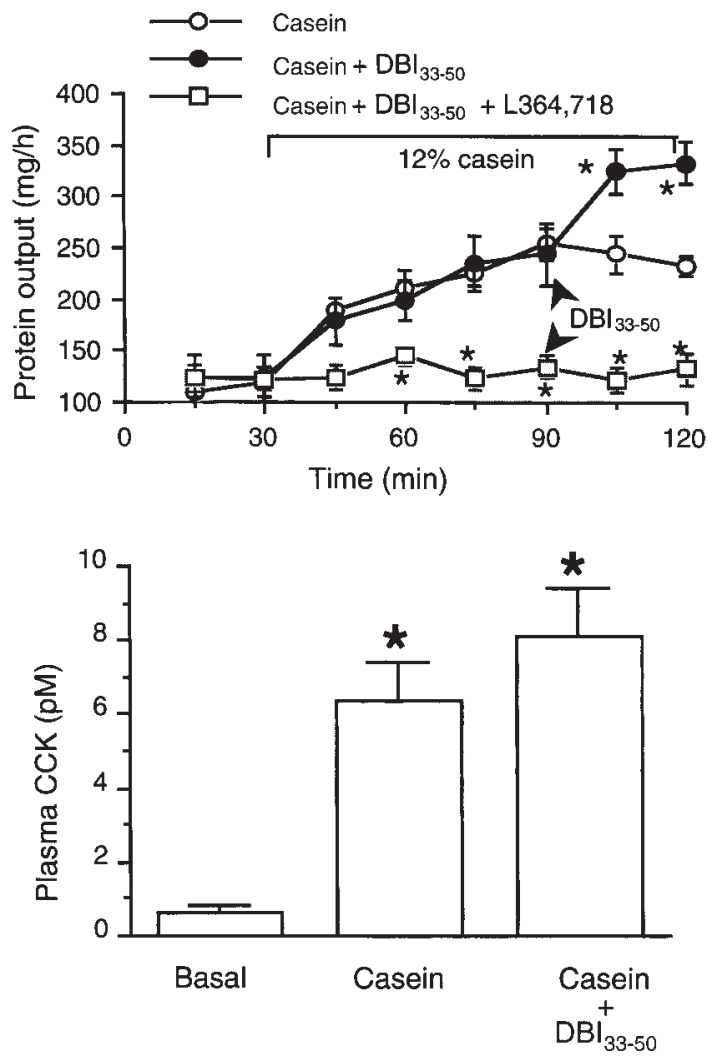

Figure 3

Effect of intraduodenal administration of $\mathrm{DBI}_{33-50}$ on pancreatic protein secretion and plasma CCK levels with a background intraduodenal infusion of $12 \%$ casein. Values are means \pm SE for 6 rats in each group. Pancreatic outputs were compared using ANOVA (total degrees of freedom, 143; $F=150.60 ;{ }^{*} P<0.01$; significant difference between casein and casein plus $\mathrm{DBl}_{33-50}$ ). Plasma CCK levels were compared using Student's $t$ test.

ed in a gamma counter. The RIA has minimal detectable concentration of $1 \mathrm{pM}, \mathrm{IC}_{50}$ of $7 \mathrm{pM}$ for porcine $\mathrm{DBI}_{33-50}$ and $9 \mathrm{pM}$ for $\mathrm{DBI}_{1-86}$, respectively. The intra-assay and interassay variations were 5 and $12 \%$, respectively.

Immunoneutralization of luminal DBI. To demonstrate that DBI mediates the release of endogenous CCK, we used 2 experimental techniques that are known to elevate plasma CCK: diversion of bile-pancreatic juice secretion and intraduodenal infusion of peptone. After a 30-minute basal period, intraduodenal administration of preimmune rabbit serum or the anti-DBI serum was followed with diversion of bile-pancreatic juice or infusion of peptone. The anti-DBI serum or preimmune rabbit serum $(2.5 \mathrm{~mL})$ was infused over 30 minutes. Pancreatic protein secretion and plasma CCK levels were monitored as described previously. To demonstrate the specificity of anti-DBI antiserum for inhibiting endogenous CCK release, a separate group of rats received an intraduodenal infusion of maltose $(300 \mathrm{mM} ; 3 \mathrm{~mL} / \mathrm{h})$, either with or without concurrent intraduodenal administration of anti-DBI serum. Intraduodenal maltose is known to stimulate pancreatic enzyme secretion by a CCK-independent mechanism (15).
Statistical analysis. Results were expressed as the mean \pm SE. For plasma CCK levels and luminal DBI immunoreactivities in the intestinal washings, data were compared by paired Student's $t$ test, and significance was accepted at the $5 \%$ level. For pancreatic protein secretion studies the multivariate analysis of variance was used to evaluate the effects of repeated measurements over time, treatment effects, and the interaction between them. The Student-Newman-Keuls multiple comparison tests were used for post-ANOVA comparison of the means. Statistical significance was accepted at 0.05 .

\section{Results}

Effects of DBI $33-50$ on plasma CCK levels and pancreatic secretion. Diversion of bile-pancreatic juice from the intestine caused marked increases in plasma CCK levels and pancreatic secretion. After a 5-hour diversion, CCK levels and pancreatic secretion gradually returned to basal levels as a result of the reduction in the concentration of luminal CCK-RP (12). This period is optimal for testing the ability of DBI to stimulate CCK secretion. Five hours after diversion of bile-pancreatic juice, the pancreatic protein output was stable, averaging $120 \pm 5 \mathrm{mg} / \mathrm{h}$. Basal plasma CCK was $0.5 \pm 0.1 \mathrm{pM}$. Intraduodenal administration of $\mathrm{DBI}_{33-50}(50 \mu \mathrm{g})$ induced a significant increase in pancreatic protein output accompanied by an increase of plasma CCK to $6.4 \mathrm{pM}$. Intravenous administration of the CCK-A receptor antagonist L364,718 completely abolished the increase in protein secretion, suggesting that pancreatic protein secretion stimulated by $\mathrm{DBI}_{33-50}$ is mediated by the secretion of CCK (Figure 1). In separate studies, intraduodenal infusion of $\mathrm{DBI}_{33-50}$ induced a dose-dependent increase in pancreatic protein and plasma CCK release (Figure 2). An intraduodenal injection of $500 \mu \mathrm{g} \mathrm{DBI}_{33-50}$ induced the maximal response: a plasma CCK level of $8.7 \mathrm{pM}$. The administration of L364,718 completely abolished the increase in pancreatic secretion (Figure 2). These observations support the hypothesis that DBI is a CCK-RP and indicate that the pancreatic secretion responses observed after administration of DBI are mediated by the release of endogenous CCK.

In separate studies we showed that intraduodenal infusion of $12 \%$ casein $(3 \mathrm{~mL} / \mathrm{h})$ produced a sustained increase in protein output. At 30 minutes after casein infusion, the mean protein output was $216 \mathrm{mg} / \mathrm{h}$, a $71 \%$ increase above basal levels. The increase in protein secretion was blocked by the CCK-receptor antagonist L364,718 (Figure 3). Intraluminal infusion of $10 \mu \mathrm{g}$ $\mathrm{DBI}_{33-50}$ with a background duodenal infusion of casein induced a secretion rate significantly greater than that observed with casein alone (Figure 4). The pancreatic protein output after DBI infusion was $320 \mathrm{mg} / \mathrm{h}$, which represented an additional $62 \%$ increase in protein secretion. The CCK receptor antagonist L364,718 completely blocked this increase. Plasma CCK levels increased from $6.4 \mathrm{pM}$ after the casein infusion to $8.2 \mathrm{pM}$ after the $\mathrm{DBI}_{33-50}$ infusion $(P<0.05)$. 


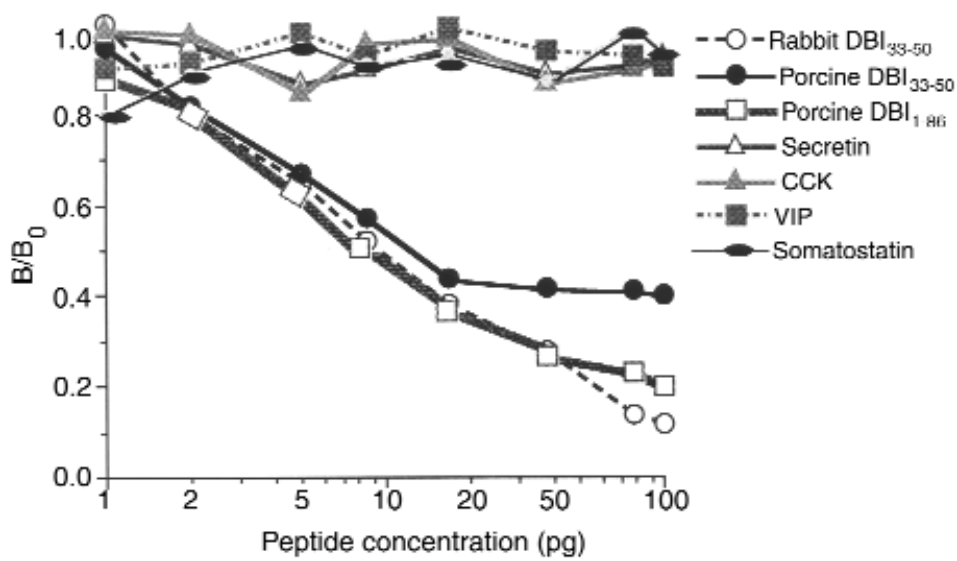

Figure 4

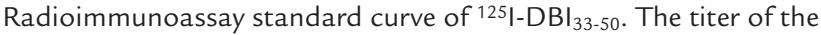
antiserum checked with ${ }^{125} \mathrm{I}_{-} \mathrm{DBI}_{33-50}$ was $1: 40,000$. It shows complete cross-reactivity with porcine $\mathrm{DBI}_{1-86}$ but has no cross-reactivity with secretin, CCK-8, VIP, or somatostatin.

Luminal secretion of DBI. To perform RIA and immunoneutralization studies, we generated an antibody in rabbits directed against porcine $\mathrm{DBI}_{33-50}$. The standard binding curve of this antibody is shown in Figure 4. The titer of the antiserum was 1:40,000, and the $\mathrm{IC}_{50}$ was 7 pg. This antibody showed complete cross-reactivity with $\mathrm{DBI}_{33-50}$ and $\mathrm{DBI}_{1-86}$ but demonstrated no cross-reactivity with secretin, CCK, VIP, or somatostatin. Using this RIA we measured DBI immunoreactivities in the intestinal washings. In vitro studies showed that the recoveries of porcine $\mathrm{DBI}_{33-50}$ and $\mathrm{DBI}_{1-86}$ from intestinal washings were $84 \pm 12 \%$ and $87 \pm 7 \%$, respectively. To demonstrate that DBI is released during diversion of bile-pancreatic juice, we measured luminal DBIimmunoreactivity at different time points after the diversion. In our experimental conditions (12), at 2 hours after diversion of bile-pancreatic juice, there was a maximal increase of plasma CCK to $8.4 \mathrm{pM}$. This increase was accompanied by an increase in luminal DBI immunoreactivity from a basal level of $1.8 \mathrm{ng} / \mathrm{h}$ to $8 \mathrm{ng} / \mathrm{h}$. At 5 hours after bile-pancreatic juice diversion, plasma CCK returned to basal levels and luminal DBI immunoreactivity fell to $2.1 \mathrm{ng} / \mathrm{h}$ (Figure 5). Peptone infusion at this time point induced an increase of DBI to $11.5 \mathrm{ng} / \mathrm{h}$, which was accompanied by an increase of plasma CCK to $6.8 \mathrm{pM}$. Luminal DBI immunoreactivity appeared to parallel changes in plasma CCK levels during diversion of bile-pancreatic juice and during intraduodenal administration of peptone.

Immunoneutralization studies. To demonstrate that $\mathrm{DBI}$ is responsible for the release of endogenous CCK, we used 2 experimental techniques that are known to elevate plasma CCK levels: diversion of bile-pancreatic juice secretion and intraduodenal peptone infusion. Rabbit anti-DBI antiserum or preimmune serum $(2.5 \mathrm{~mL}$ over 30 minutes) was administered intraduodenally 15 minutes before diversion of bile-pancreatic juice or nutrient stimulation. Figure 6 shows that diversion of bile-pancreatic juice from the duodenum resulted in a rapid and sustained increase in protein output. At 60 minutes after diversion of bile-pancreatic juice, the mean protein output was 270 $\mathrm{mg} / \mathrm{h}$, which represented a 2 -fold increase above basal levels. The plasma CCK level was $8.8 \mathrm{pM}$. Administration of anti-DBI antiserum ( $2.5 \mathrm{~mL}$ over 30 minutes), but not preimmune rabbit serum, completely abolished protein secretion and CCK release in response to the diversion of bile-pancreatic juice. This response suggests that DBI is involved in mediating endogenous CCK release during bile-pancreatic juice diversion.

In separate experiments we showed that duodenal infusion of peptone produced a 2 -fold increase of protein secretion over basal levels and increased plasma CCK levels to $7.8 \mathrm{pM}$. Intraduodenal administration of rabbit anti-DBI antiserum ( $2.5 \mathrm{~mL}$ over 30 minutes) significantly inhibited protein secretion and plasma CCK responses to peptone stimulation (Figure 7), indicating that DBI mediates the release of CCK in response to peptone stimulation. In addition, we showed that intraduodenal administration of rabbit anti-DBI antiserum abolished protein secretion and plasma CCK responses to stimulation by intraduodenal administra-
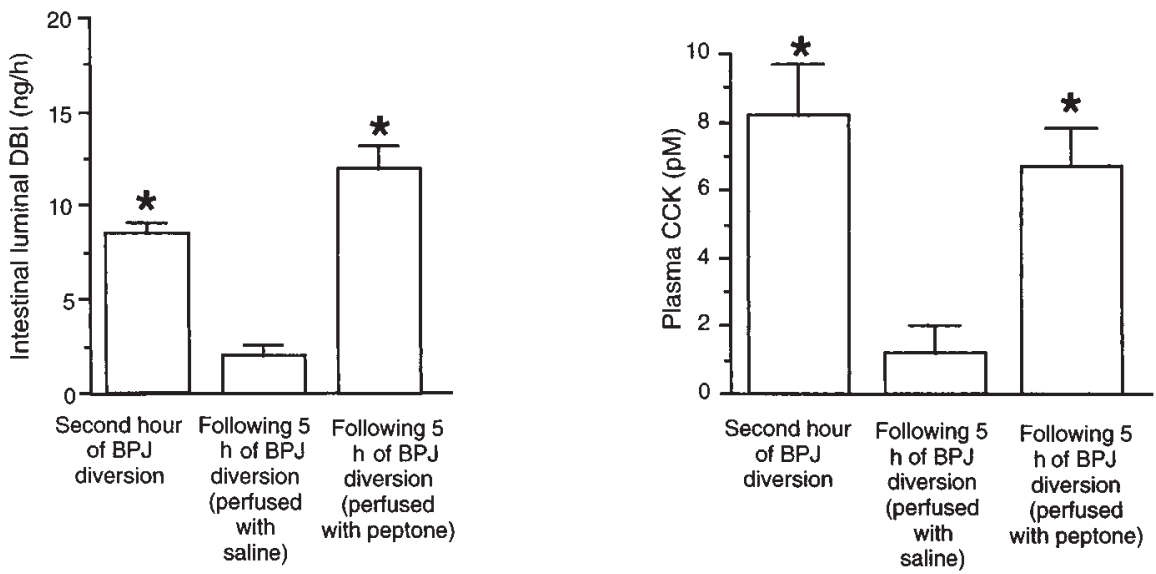

Figure 5

Intestinal DBI immunoreactivity and plasma CCK levels during BPJ diversion and during peptone stimulation. Values are means \pm SE for 6 rats in each group. ${ }^{*} P<0.01$ by Student's $t$ test. 

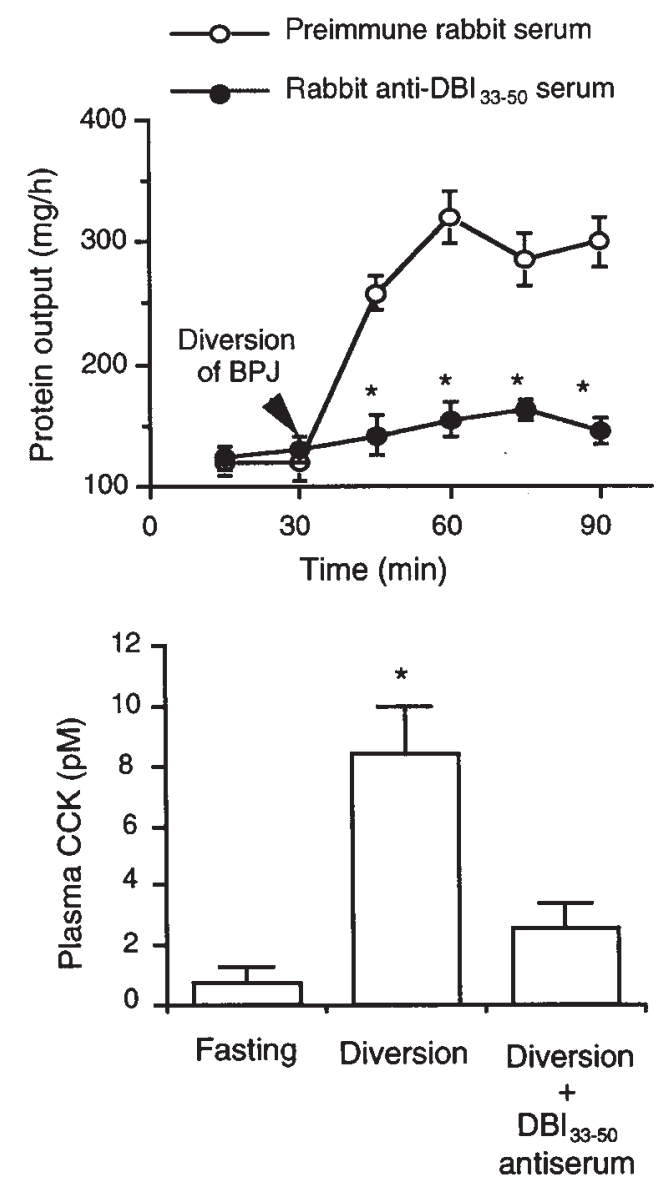

Figure 6

Effect of rabbit anti-DB| $\left.\right|_{33-50}$ antiserum on pancreatic secretion and plasma CCK stimulated by diversion of BPJ. Values are means $\pm \mathrm{SE}$ for 6 rats in each group. Pancreatic protein outputs were compared using ANOVA (total degrees of freedom, 71; $F=314.06$; ${ }^{*} P<0.01$ ). Plasma CCK levels were compared using Student's $t$ test, ${ }^{*} P<0.01$.

tion of $200 \mathrm{ng}$ of porcine $\mathrm{DBI}_{1-86}$ synthesized as reported previously (10). This suggests that the anti-DBI antiserum used in our study is capable of recognizing the full DBI molecule. To demonstrate the specificity of the anti-DBI antiserum to inhibit CCK-mediated pancreatic secretion, we showed that intraduodenal infusion of $300 \mathrm{mM}$ maltose $(3 \mathrm{~mL} / \mathrm{h})$ evoked a 2 -fold increase of pancreatic protein output, but did not stimulate plasma CCK levels (15). Pretreatment with intraduodenal administration of anti-DBI antiserum had no effect on maltose-induced pancreatic secretion (Figure 8).

\section{Discussion}

CCK is one of the most important hormonal regulators of the digestive process. CCK is produced primarily by endocrine cells in the mucosa of the proximal small intestine, and it is secreted into the circulation in response to protein (16) and fats (14). The manner by which food affects the secretion of CCK is largely unknown. The apical surface of the CCK cell is oriented toward the lumen of the gut, and the secretory gran- ules that contain CCK are concentrated basolaterally (17). This structure enables nutrients and other factors within the intestinal lumen to interact with the microvillus-like processes of the CCK cell, which, in turn, stimulates the secretion of CCK from the basal surface. Although recent studies indicate that phenylalanine may stimulate CCK secretion from STC-1 cells (18), it is not apparent if nutrients can directly stimulate CCK secretion from cells in the intestine that contain CCK. Experimental evidence suggests that CCK secretion may be mediated by a trypsin-sensitive CCK$\mathrm{RP}$ that is secreted into the intestinal lumen. At least 3 luminally secreted peptides have been shown to stimulate CCK release when administered intraduodenally: monitor peptide purified from rat pancreatic juice, luminal CCK-releasing factor obtained from perfusion of the rat intestine after prolonged ( 6 days) diversion of bile-pancreatic juice, and CCK-RP purified from porcine and rat intestinal mucosa. It is not known, however, if one or more of these luminally secreted peptides are responsible for feedback regulation of pancreatic secretion and postprandial CCK release.

Diversion of bile-pancreatic juice establishes a critical experimental condition in that it prevents pancreatic proteases from entering the intestine; thus, infused protease-sensitive peptides are not rapidly inactivated. The removal of proteases from the rat proximal intestine also induces CCK release, resulting in a high background level of plasma CCK and pancreatic protein secretion. This hampers the detection of changes in pancreatic protein output and plasma CCK levels in response to intestinal infusion of putative CCK-RPs. Some investigators have used taurocholate to lower the high plasma CCK levels after diversion of bile-pancreatic juice to test the activity of putative CCK-RPs (19) because taurocholate inhibits "spontaneous" CCK release and pancreatic protein secretion. However, a previous study has clearly shown that bile induced a negative feedback regulation of CCK release and inhibited postprandial CCK secretion (20). Furthermore, we have shown that the inhibitory action of bile is mediated by inhibiting the ability of CCK-RP (DBI) to release CCK (20). Therefore, it is inappropriate to test the activity of CCK-RP after intraduodenal infusion of taurocholate in the rat with bile-pancreatic juice diversion. We have shown previously that diversion of bile-pancreatic juice from the intestine resulted in a marked increase in plasma CCK levels and pancreatic secretion (12). These increases gradually returned to basal levels after a 5-hour diversion as a result of a reduction in luminal CCK-RP (12).

For practical reasons, we used $\mathrm{DBI}_{33-50}$ to investigate the ability of a luminal CCK-RP to release CCK. $\mathrm{DBI}_{1-}$ 86 synthesis is expensive and thus impractical for whole animal studies. $\mathrm{DBI}_{33-50}$ is a product of tryptic digestion of the DBI peptide. It has been detected as a naturally occurring peptide in rat brain homogenates (21) and intestinal mucosa (22). This peptide is capable of eliciting proconflict response in rats (23), but it is 100 times less potent compared with the full peptide in 

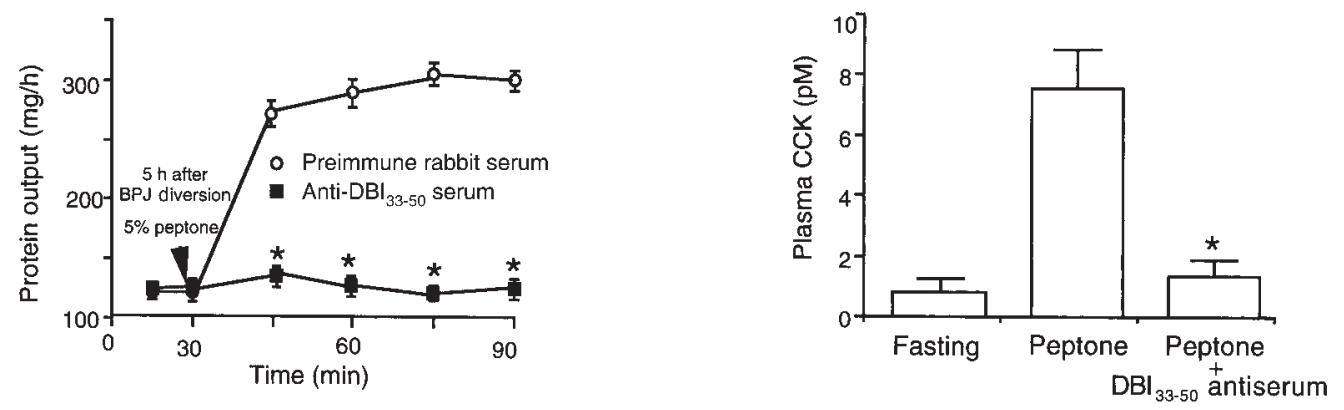

Figure 7

Effect of rabbit anti-DBI ${ }_{33-50}$ antiserum on pancreatic secretion and plasma CCK stimulated by duodenal infusion of peptone. Values are means \pm SE for 6 rats in each group. Pancreatic protein outputs were compared using ANOVA (total degrees of freedom, 71; $F=$ 267.03; $\left.{ }^{*} P<0.01\right)$. Plasma CCK levels were compared using Student's $t$ test, ${ }^{*} P<0.01$.

stimulating CCK release (10). Trypsin cleaves the internal bonds at the lysine or arginine residues. Therefore, even though $\mathrm{DBI}_{33-50}$ no longer contains a lysine residue, it is still sensitive to trypsin because it contains arginine-proline residues.

In the presence of active proteases such as trypsin, CCK-RP is cleaved and inactivated. However, dietary protein in the intestine competes for trypsin and prevents protease-induced feedback inhibition of CCK release. The resulting increase of active CCK-RP induces the release of CCK and stimulates pancreatic enzyme secretion, which has been proposed to be the major mechanism by which intact proteins stimulate CCK release in rats (9). To further evaluate if proteins such as casein in the intestine could prevent the inactivation of $\mathrm{DBI}_{33-50}$ by proteases, we investigated the action of $\mathrm{DBI}_{33-50}$ in the presence of casein in a rat model without bile-pancreatic juice diversion. Our studies demonstrated that under this experimental condition, intraduodenal infusion of $\mathrm{DBI}_{33-50}$ induced a significant increase in plasma CCK levels. These observations suggest that that DBI can stimulate the release of CCK postprandially, even in the presence of bile-pancreatic juice in the intestine, providing there is sufficient protein to bind proteolytic enzymes in the lumen.

Another criterion to support the role of DBI as a physiologic regulator of CCK secretion is evidence that luminal DBI concentrations parallel plasma CCK levels under various physiologic conditions. We used an antibody against the porcine $\mathrm{DBI}_{33-50}$ fragment for a RIA to quantify the luminal DBI concentration. The 18-amino acid peptide fragment (octadecaneuropeptide) is present in DBI molecules of all species (21) and has been shown to displace labeled DBI-like ligand from specific binding sites $(21,23)$. Furthermore, we demonstrated that the antiserum generated against $\mathrm{DBI}_{33-50}$ has complete crossreactivity with porcine $\mathrm{DBI}_{1-86}$ and $\mathrm{DBI}_{33-50}$. Therefore, it is reasonable to use an antiserum generated against $\mathrm{DBI}_{33-50}$ to perform RIA and immunoneutralization studies. We detected low concentrations of DBI in the gut lumen under basal conditions. Two hours after diversion of bile-pancreatic juice, a 4-fold increase in DBI immunoreactivity was observed. This was accom- panied by an increase in plasma CCK levels from 0.7 to $8.4 \mathrm{pM}$. The level of luminal DBI immunoreactivities after a 2-hour diversion of bile pancreatic juice could be reproduced by intraduodenal infusion of $50-100 \mu \mathrm{g}$ of $\mathrm{DBI}_{33-50}$, which in turn stimulated an increase of plasma CCK levels to 6-8 pM (unpublished data). The spontaneous secretion of DBI under basal conditions appears to be mediated by a cholinergic pathway (7). However, the luminal concentration of DBI is low because of trypsin inactivation. Diversion of bile-pancreatic juice permits accumulation of luminal DBI, which produces an increase of circulating CCK. Because DBI exists in more than one molecular form, it is important to characterize the molecular form(s) that is secreted into the intestine. Our preliminary studies indicated that the majority of the DBI immunoreactivities in the intestinal washings were found in fractions representing molecular weights between 7,500 and 10,000. This suggests that the main molecular form of DBI secreted into the lumen is likely to be $\mathrm{DBI}_{1-86}$.

Previously, we have used a donor-recipient rat intestine perfusion model to quantify CCK-RP secretion

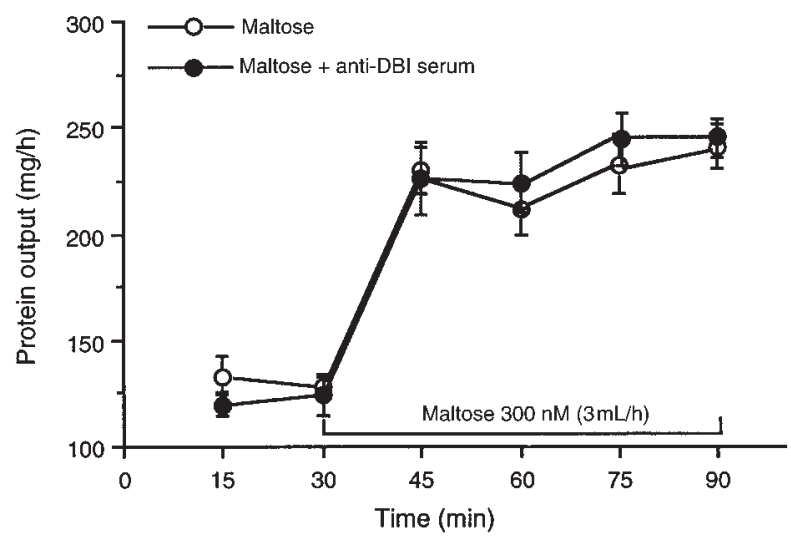

Figure 8

Effect of rabbit anti-DB| ${ }_{33-50}$ antiserum on pancreatic secretion and plasma CCK stimulated by duodenal infusion of maltose. Values are means $\pm \mathrm{SE}$ for 6 rats in each group. Administration of anti-DBI $33-50$ antiserum did not alter the pancreatic response to maltose. (ANOVA, total degrees of freedom, $71 ; F=280.70)$. 
and have shown that after a 5-hour diversion of bile-pancreatic juice, the intestinal perfusates collected from the donor rat did not contain significant amounts of CCK-RP (9). This is consistent with our current observation: after a 5-hour diversion of bile-pancreatic juice, luminal DBI immunoreactivity fell to basal levels and plasma CCK fell to $0.6 \mathrm{pM}$. This lowering of luminal DBI concentrations and plasma CCK levels may be mediated by the release of somatostatin stimulated by increased plasma CCK secretion during bile-pancreatic juice diversion, which in turn inhibits CCK-RP secretion (12). Furthermore, luminal nutrients, such as peptone-stimulated luminal DBI secretion. After a 5-hour diversion of bile-pancreatic juice, infusion of peptone resulted in a 5-fold increase in luminal DBI immunoreactivity. These data are consistent with our previous observation that peptone in the duodenum acts by stimulating the luminal secretion of CCK-RP, which in turn stimulates CCK release $(9,14)$. DBI immunostaining and in situ hybridization studies in the rat confirmed that it is widely distributed in the villi of the upper intestine (22). The location of CCK cells in the intestinal mucosa $(17,24)$, with their apical surfaces exposed to the lumen of the gut, provides an opportunity for DBI to interact directly with the CCK cell to stimulate hormone secretion. We recently demonstrated that $\mathrm{DBI}_{33-50}$ acted directly on a murine tumor cell line, STC-1, that contains CCK, eliciting $\mathrm{Ca}^{2+}$ oscillations through the voltage-dependent L-type $\mathrm{Ca}^{2+}$ channel and inducing CCK secretion (25).

To provide conclusive evidence that DBI mediates CCK secretion during diversion of bile-pancreatic juice and postprandial secretion in the rat, we performed immunoneutralization studies using an antibody developed against $\mathrm{DBI}_{33-50}$. Our studies showed that intraduodenal administration of anti-DBI antiserum, but not preimmune serum, abolished the physiologic responses of CCK release and pancreatic secretion during bile-pancreatic juice diversion and during intraduodenal peptone stimulation. In contrast, intravenous infusion of anti-DBI antibody did not affect these physiologic responses (data not shown). We also demonstrated that intraduodenal infusion of the antiDBI antiserum prevented the increase in protein secretion and plasma CCK levels stimulated by $\mathrm{DBI}_{1-86}$, indicating that the antiserum used in this study was capable of recognizing the gut DBI peptide. The action of anti-DBI antiserum is specific for inhibiting CCKmediated pancreatic secretion, as shown by the lack of effect of anti-DBI antiserum on pancreatic secretion evoked by intraduodenal administration of maltose, which stimulates pancreatic secretion independent of CCK (15) but is mediated by serotonin (5-HT) sensory vagal afferent pathways $(26,27)$. These observations further support the hypothesis that luminally released DBI mediates the feedback regulation of pancreatic secretion and the postprandial release of CCK.

The physiological control of pancreatic enzyme secretion and CCK release may differ between anes- thetized and conscious rats. To confirm that luminal DBI also mediates CCK release in the conscious state, we performed bile-pancreatic juice diversion studies in conscious rats as described previously (28). Our preliminary studies indicated that similar to the observation made in anesthetized rats, diversion of bile pancreatic juice resulted in a significant increase in plasma CCK levels, from a basal level of $1.9 \pm 0.4$ pM to $8.9 \pm$ $2.3 \mathrm{pM}$. Intraduodenal administration of rabbit antiDBI serum ( $2.5 \mathrm{~mL} / 30 \mathrm{~min})$ abolished the increase in plasma CCK levels after diversion of bile pancreatic juice (unpublished data). This suggests that luminal DBI also plays a key role in the mediation of CCK release in conscious rats.

Apart from DBI, there are a number of luminally secreted peptides that have been shown to stimulate CCK release when administered intraduodenally. The pancreatic monitor peptide is found exclusively in zymogen granules of pancreatic acinar cells (29) and is acid stable, heat resistant, and has a molecular weight of approximately $6.50 \mathrm{kDa}$. This peptide has a partial sequence homology to epidermal growth factor and has been shown to stimulate CCK release (30) and pancreatic enzyme secretion (31) when administered into the rat proximal intestine by an atropine-insensitive mechanism (31). The physiologic function of this peptide is not certain; it is only present in pancreatic juice, which proves that it is not involved in the feedback regulation of pancreatic secretion as first reported by Green and Lyman (2). Spannagel and colleagues (11) recently purified another luminal CCK-releasing factor from the duodenal secretion of rats. Partially purified fractions of this peptide increased the release of CCK and stimulated pancreatic secretion of fluid and protein. Amino acid sequencing and mass spectral analysis revealed that this peptide is composed of 70-75 amino acids with no homology to DBI. Currently, its physiologic function remains unclear. Our preliminary studies demonstrated that although intraduodenal administration of antiserum directed against $\mathrm{DBI}_{33-50}$ abolished pancreatic secretion in response to diversion of bile-pancreatic juice or peptone stimulation, it had no effect on pancreatic secretion stimulated by this novel luminal CCK-releasing factor (unpublished data). Our studies, however, do not rule out the participation of this peptide in the mediation of the feedback regulation of pancreatic secretion or the postprandial release of CCK.

In summary, we have demonstrated the following: (a) intraduodenal administration of $\mathrm{DBI}_{33-50}$ dose-dependently increased pancreatic secretion and plasma CCK levels; (b) DBI immunoreactivities in the intestinal lumen paralleled changes in plasma CCK levels during diversion of bile-pancreatic juice and during intraduodenal peptone stimulation; and (c) intraduodenal administration of anti-DBI antisera abolished pancreatic secretion and release of CCK during bile-pancreatic juice diversion and peptone stimulation. These observations strongly support the hypothesis that DBI 
is the physiologic CCK-releasing peptide responsible for the feedback regulation of pancreatic secretion and the postprandial release of CCK in the rat.

\section{Acknowledgments}

This work was supported by the National Institute of Diabetes and Digestive and Kidney Diseases Grants R01-DK 32830 and 5P30-DK 34933.

1. Lyman, R.L., and Lepkovsky, S. 1957. The effect of raw soybean meal and trypsin inhibitor diets on pancreatic enzyme secretion in the rat. J. Nutr. 62:269-284.

2. Green, G.M., and Lyman, R.L. 1972. Feedback regulation of pancreatic enzyme secretion as a mechanism for trypsin inhibitor-induced hypersecretion in rats. Proc. Soc. Exp. Biol. Med. 140:6-12.

3. Chernick, S.S., Lepkovsky, S., and Chaikoff, I.L. 1948. A dietary factor regulating the enzyme content of the pancreas: changes induced in size and proteolytic activity of the chick pancreas by the ingestion of raw soybean meal. Am. J. Physiol. 155:33-41.

4. Corring, T. 1973. Mecanisme de la secretion pancreatique exocrine chez le porc: regulation par retroinhibition. Ann. Biol. Anim. Biochim. Biophys. 13:755-756.

5. Owyang, C., Louie, D.S., and Tatum, D. 1986. Feedback regulation of pancreatic enzyme secretion. Suppression of cholecystokinin release by trypsin. J. Clin. Invest. 77:2042-2047.

6. Louie, D.S., May, D., Miller, P., and Owyang, C. 1986. Cholecystokinin mediates feedback regulation of pancreatic enzyme secretion in rats. $\mathrm{Am}$. J. Physiol. 250:G252-G259.

7. Lu, L., Louie, D., and Owyang, C. 1989. A cholecystokinin releasing peptide mediates feedback regulation of pancreatic secretion. Am. J. Physiol. 256:G430-G435.

8. Miyasaka, K., Guan, D., Liddle, R.A., and Green, G.M. 1989. Feedback regulation of trypsin: evidence for intraluminal CCK-releasing peptide. Am. J. Physiol. 257:G175-G181.

9. Li, Y., and Owyang, C. 1996. Peptone stimulates CCK-releasing peptide secretion by activating intestinal submucosal cholinergic neurons. J. Clin. Invest. 96:1463-1470.

10. Herzig, K.H., et al. 1996. Diazepam binding inhibitor is a potent cholecystokinin-releasing peptide in the intestine. Proc. Natl. Acad. Sci. USA. 93:7927-7932.

11. Spannagel, A.W., et al. 1996. Purification and characterization of a luminal cholecystokinin-releasing factor from rat intestinal secretion. Proc. Natl. Acad. Sci. USA. 93:4415-4420.

12. Li, Y., Hao, Y., and Owyang, C. 1995. Evidence for autoregulation of cholecystokinin (CCK) secretion during diversion of bile-pancreatic juice in rats. Gastroenterology. 109:231-238.

13. Li, Y., and Owyang, C. 1994. Endogenous cholecystokinin stimulated pancreatic enzyme secretion via a vagal afferent pathway. Gastroenterology. 107:525-531.

14. Li, Y., Hao, Y.B., and Owyang, C. 1996. CCK-releasing peptide mediates oleic acid stimulated secretion of CCK: involvement of intestinal sub- mucosal cholinergic neurons. Regul. Pept. 64:110. (Abstr.)

15. Li, Y., and Owyang, C. 1996. Pancreatic secretion evoked by cholecystokinin and non-cholecystokinin dependent duodenal stimuli via vagal afferent fibers in rat. J. Physiol. (Lond.). 493:773-782.

16. Liddle, R.A., Green, G.M., Conrad, C.K., and Williams, J.A. 1986. Proteins but not amino acids, carbohydrates, or fats stimulate cholecystokinin secretion in the rat. Am. J. Physiol. 251:G243-G248.

17. Buchan, A.M.J., et al. 1978. Electron immunohistochemical evidence for the human intestinal I cell as the source of CCK. Gut. 19:403-407.

18. Mangel, A.W., et al. 1995. Phenylalanine-stimulated secretion of cholecystokinin is calcium dependent. Am. J. Physiol. 268:G90-G94.

19. Difu, G., Ohta, H., Tawil, T., Liddle, R.A., and Green, G.M. 1995. CCKreleasing activity of rat intestinal secretion: effect of atropine and comparison with monitor peptide. Pancreas. 5:677-684

20. Li, Y., Hao, Y.B., and Owyang, C. 1997. Bile-induced feedback inhibition of CCK release is mediated by inhibition of the action of CCK-releasing peptide (diazepam-binding inhibitor). Gastroenterology. 112:458. (Abstr.)

21. Ferrero, P., Santi, M.R., Conti-Tronconi, B., Costa, E., and Guidotti, A. 1986. Study of an octadecaneuropeptide derived from diazepam bindng inhibitor (DBI) biological activity and presence in rat brain. Proc. Natl. Acad. Sci. USA. 83:827-831.

22. Steyaert, H., et al. 1991. Distribution and characterization of endogenous benzodiazepine receptor ligand (endozepine)-like peptides in the rat gastrointestinal tract. Endocrinology. 129:2101-2109.

23. Kavaliers, M., and Hirst, M. 1986. An octadecaneuropeptide (ODN) delivered from diazepam binding inhibitor increases aggressive interactions in mice. Brain Res. 383:343-349.

24. Polak, J.A., et al. 1975. Identification of cholecystokinin-secreting cells. Lancet. 2:1016-1021.

25. Yoshida, H., Tsunoda, Y., and Owyang, C. 1999. Diazepam-binding inhibitor 33-50 elicits $\mathrm{Ca}^{2+}$ oscillation and CCK secretion in STC-1 cells via L-type $\mathrm{Ca}^{2+}$ channels. Am. J. Physiol. 276:G694-G702.

26. Li, Y., and Owyang, C. 1994. Non-CCK dependent duodenal stimuli stimulate pancreatic enzyme secretion via gastroduodenal vagal afferent fibers: mediation via 5HT sensory pathways. 1994. Pancreas. 9:795. (Abstr.)

27. Zhu, J.X., Owyang, C., and Li, Y. 1998. Sensitivity of vagal mucosal afferents to serotonin and its role in the mediation of non-CCK-stimulated pancreatic secretion. Dig. Dis. Sci. 43:A30. (Abstr)

28. Li, Y., and Owyang, C. 1996. Pancreatic secretion evoked by cholecystokinin and non-cholecystokinin-dependent duodenal stimuli via vagal afferent fibers in the rat. J. Physiol (Lond.). 494:773-782.

29. Iwai, K., et al. 1987. Purification and sequencing of a trypsin-sensitive cholecystokinin-releasing peptide from rat pancreatic juice. J. Biol. Chem. 262:8956-8959.

30. Iwai, K., Fukuka, S.I., Fushiki, T., Kodaira, T., and Ikei, N. 1986. Elevation of plasma CCK concentration after intestinal administration of a pancreatic enzyme secretion-stimulating peptide purified from rat bilepancreatic juice: analysis with $\mathrm{N}$-terminal region specific radioimmunoassay. Biochem. Biophys. Res. Commun. 136:701-706.

31. Fushiki, T., Fukuka, S.I., and Iwai, K. 1984. Stimulatory effect of an endogenous peptide in rat pancreatic juice on pancreatic enzyme secretion in the presence of atropine: evidence for different mode of action of stimulation from exogenous trypsin inhibitors. Biochem. Biophys. Res. Commun. 118:532-537. 